\title{
Der rechtliche Status der Europäischen Konvention zum Schutze der Menschenrechte und Grundfreiheiten und der Rechtsprechung des Europäischen Gerichtshofs für Menschenrechte sowie deren Rolle bei der Gestaltung des russischen Strafverfahrens
}

\section{Summary}

Russia's accession to the Council of Europe (1996) and ratification of the European Convention for the Protection of Human Rights and Fundamental Freedoms (ECHR) in 1998 symbolize a new stage of development for the protection of human rights in Russia. In the first part of the paper, the main stages of development that served as conditions for the application of international mechanisms within the domestic legal system are discussed. After fifteen years of the application of the Convention, relevant questions regarding the status of the ECHR and the case law of the European Court of Human Rights (ECtHR) in the hierarchy of legal sources of Russian law remain unanswered. In the second part of this article, these controversies are analyzed. Since 1998, Russia has been under the jurisdiction of the ECtHR. The case law of the ECtHR has undoubtedly had a significant impact on both the development of the criminal procedural legislation and the national legal practice with its consistent humanization and internationalization. In addition, an opportunity to protect the rights within the national legal system using the guarantees contained in the Convention appeared. The third part of the article is therefore devoted to the analysis of the impact of the legal provisions of the ECHR and of ECtHR case law on some aspects of Russian criminal procedure.

\section{Résumé}

L'adhésion de la Russie au Conseil de l'Europe (1996) et la ratification de la Convention européenne de sauvegarde des droits de l'homme et des libertés fondamentales (CEDH) en 1998 symbolisent la nouvelle phase de développement pour la protection des droits de l'homme en Russie. La première partie de l'article décrit les principales phases de développement qui ont représenté les conditions pour l'application des mécanismes internationaux dans l'ordre juridique interne. Après 15 années d'application de la Convention, d'importantes questions n'ont pas encore reçu de réponse par rapport au statut de la CEDH et de la jurisprudence de la Cour européenne des droits de l'homme (CtEDH) dans la hiérarchie des sources du droit russe. Dans la deuxième partie de cet article, l'Auteur envisage ce débat. Depuis 1998, la Russie est soumise à la juridiction de la CtEDH. La jurisprudence de la CtEDH a sans doute un impact significatif sur le développement de la législation processuelle pénale aussi bien que sur la pratique du droit national, grâce à son æuvre d'humanisation et d'internationalisation. Par ailleurs, 
il est aussi possible de protéger les droits dans le système juridique national à travers les garanties contenues dans la Convention. La troisième partie de l'article est consacrée à l'analyse de l'impact des dispositions normatives de la CEDH et de la jurisprudence de la CtEDH sur divers aspects de la procédure pénale russe.

\section{„Steter Tropfen höhlt den Stein“}

Der Beitritt Russlands zum Europarat $(1996)^{1}$ und die Ratifizierung der Europäischen Konvention zum Schutz der Menschenrechte und Grundfreiheiten (EMRK) ${ }^{2}$ im Jahre 1998 symbolisieren den neuen Entwicklungsabschnitt für den Schutz der Menschenrechte in Russland. Im ersten Teil des Beitrags wird auf die wichtigsten Entwicklungsabschnitte in der nationalen Rechtsordnung eingegangen, die als Voraussetzungen für die Anwendung der internationalen Mechanismen dienten (I.). Nach 15 Jahren der Geltung der Konvention bleiben immer noch offene Fragen bezüglich des Status der Konvention und der Rechtsprechung des Europäischen Gerichtshofs für Menschenrechte $(\mathrm{EGMR})^{3}$ in der Hierarchie der Rechtsquellen des russischen Rechts. Diese Fragen werden im zweiten Teil dieses Beitrags analysiert (II.). Seit 1998 unterliegt Russland der Zuständigkeit des EGMR, dessen Rechtsprechung unumstritten einen bedeutsamen Einfluss sowohl auf die Entwicklung der strafprozessualen Gesetzgebung als auch auf die nationale Rechtspraxis und deren konsequente Humanisierung und Internationalisierung hat. ${ }^{4}$ Darüber hinaus entstand eine Möglichkeit, die Rechte des nationalen Rechtssystems unter Einsatz der in der Konvention enthaltenen Garantien zu schützen. Der dritte Teil des Aufsatzes ist daher der Analyse des Einflusses der Vorschriften der EMRK sowie der EGMR-Rechtsprechung auf einige Aspekte des russischen Strafverfahrens gewidmet (III.).

\section{Einführung}

Am 28. Februar 1996 trat Russland dem Europarat bei. Zum Zeitpunkt des Beitritts jedoch fehlten die notwendigen Voraussetzungen im nationalen Rechtssystem, um den europäischen Menschenrechtsstandards zu entsprechen. Die Mitgliedschaft Russlands ist daher eine gegenseitige politische Entscheidung gewesen, die unter der Bedingung getroffen wurde, dass die Russische Föderation nach dem Beitritt, insbesondere nach der Ratifizierung der Konvention zum Schutze der Menschenrechte einschließlich der Gerichtsbarkeit des EGMR, entsprechende Reformen durchführt. ${ }^{5}$ Berücksichtigt man, dass nach der sowjetischen Doktrin die Idee, die UN-Menschenrechts-Verträge direkt anzuwenden, ausgeschlossen war und die Souveränität des Staates einen klaren Vorrang

1 Föderales Gesetz "Über den Beitritt der Russischen Föderation in den Europarat" vom 23. Februar 1996, N 19-Ф3.

2 Europäische Konvention zum Schutz der Menschenrechte und Grundfreiheiten vom 4. November 1950, nachfolgend EMRK oder Konvention, http://conventions.coe.int/treaty/.

3 Europäischer Gerichtshof für Menschenrechte, nachfolgend EGMR.

4 Siehe i.E. S. Paramonova/G. Bogush, Neue Entwicklungen im Strafprozessrecht Russlands, ZStW 2013 (i.E.).

5 Föderales Gesetz „Über die Ratifizierung der EMRK“ vom 30. März 1998, N 54-FZ. 
vor den Rechten des Einzelnen hatte, war eine solche internationale Verpflichtung aus der Perspektive der Menschenrechte ein herausfordernder Neuanfang. ${ }^{6}$ Der Prozess der graduellen Schaffung von Bedingungen für die direkte Anwendung von in der Konvention verankerten Garantien in der innerstaatlichen Rechtsordnung führt zu den folgenden wichtigen Etappen zurück.

In der Phase vor dem Beitritt zum Europarat war die Verabschiedung der „Konzeption der Justizreform“ im Jahr 1991 entscheidend. ${ }^{7}$ In ihr wurden die wichtigsten Aufgaben und Entwicklungen im Justizbereich des neuen Russlands festgelegt. Unter anderem wurden in ihr progressive Ideen angekündigt, von denen das moderne Strafverfahren mittlerweile geprägt ist, wie die Differenzierung der Justizverfahren, die Wiedereinführung der Jury, die richterliche Kontrolle der prozessualen Entscheidungen aller Instanzen, welche die Menschenrechte betreffen etc. ${ }^{8}$

Der nächste wichtige Abschnitt war die Verabschiedung der neuen Verfassung der Russischen Föderation, die im Jahr 1993 durch Volksabstimmung angenommen wurde. ${ }^{9}$ Hier sollte erwähnt werden, dass die Verfassung unter dem starken Einfluss der internationalen Menschenrechtsabkommen, darunter die damals von Russland noch nicht ratifizierte Konvention zum Schutze der Menschenrechte, entworfen wurde. ${ }^{10}$ Als Ergebnis enthält die Verfassung einen kompletten Katalog der Rechte und Freiheiten des Menschen und Bürgers, einschließlich der EMRK-Garantien. ${ }^{11}$ Darunter sind die Vorschriften des zweiten, den Menschenrechten gewidmeten Kapitels der Verfassung, die einen direkten Bezug zum Strafprozess haben (Art. 46-54 Verfassung), zu nennen. Dazu zählen beispielsweise die Vorschriften über die Garantien des gerichtlichen Schutzes (Art. 46 Verfassung), das Recht auf Verhandlung der Sache vor dem Gericht und durch die Richter, die gesetzlich für sie zuständig sind (Art. 47 Abs. 1 Verfassung) etc.

Die nächste Etappe ist unmittelbar mit der Verabschiedung des neuen Strafgesetzbuches der Russischen Föderation (RF) von 1996 (UK) verbunden. ${ }^{12}$ Das neue Strafgesetzbuch hatte eine wichtige Funktion in der Übergangszeit vom Zusammenbruch der Sowjetunion zum modernen Russland. Das Strafgesetzbuch von 1996 hat die fundamentalen sozialen, wirtschaftlichen und politischen Änderungen sowohl durch Krimi-

6 L. Mälksoo Concluding Observations. Russia and European Human-Rights Law: Margins of the Margin of Appreciation. Review of Central and East European Law 37 (2012), 359.

7 Beschluss des Obersten Rates der RSFSR „Über die Konzeption der Justizreform in der RSFSR" vom 24. Oktober 1991, N1801-1.

8 S. i.E. Paramonova/Bogush (Fn. 4).

9 Verfassung der Russischen Föderation, angenommen durch Volksabstimmung vom 12. Dezember 1993; deutsche Übersetzung erstellt von M. Fincke, zu finden unter http://www.constitution.ru/de/index.htm.

10 A. Burkow, Konvenziya o zaschite prav cheloveka i praktika Evropejskogo Suda v rossijskoj pravovoj sisteme, Sravnitelnoe konstituzionnoe obozrenie, 2009, N 4, S. 121.

11 Russia's application for membership of the Council of Europe, Doc. 746318 January 1996, http://assembly.coe.int/ASP/Doc/XrefViewHTML.asp?FileID=7397\&Language=EN.

12 Strafgesetzbuch der Russischen Föderation vom 13. Juni 1996, N 63-FZ, Ugolovnij Kodeks, nachfolgend $U K$. 
nalisierung als auch durch Entkriminalisierung dem neuen Konzept nicht mehr entsprechender Verhaltensformen reflektiert. ${ }^{13}$

Dies hat unvermeidbar die Verabschiedung einer neuen Strafprozessordnung bestimmt. Die neue Strafprozessordnung RF von 2002 (UPK) ${ }^{14}$ hatte vor allem das Ziel, den neuen russischen Strafprozess von der Vernachlässigung des Schutzes der Menschenrechte gegenüber den staatlichen Interessen und von dem eindeutigen Inquisitionscharakter, der den sowjetischen Strafprozess gekennzeichnet hatte, zu entlasten. Das neue Konzept des Strafverfahrens wurde von dem Schutz des Einzelnen dienenden Prinzipien (Art. 6-19 UPK) geprägt, wie die Unschuldsvermutung, die Unantastbarkeit der Person, die Unabhängigkeit der Judikative etc. ${ }^{15}$

Soweit so gut. Doch das Hauptproblem des russischen Strafprozesses bleibt bis heute die große Kluft zwischen dem positiven Recht und der Praxis. Die menschenrechtlichen Garantien wurden - wie gesagt - sowohl in der neuen Verfassung von 1993 als auch in der neu verabschiedeten Strafprozessordnung verankert, doch finden diese, wenn überhaupt, nicht in zureichendem Maße Anwendung im tatsächlichen Strafverfahren.

Desto relevanter wird in diesem Kontext die verbindende Rolle der Konventionsgarantien und des rechtlichen Schutzsystems des EGMR. Zurzeit ist der EGMR einer der, wenn nicht der effektivste internationale Mechanismus, der das nationale Rechtssystem in Bezug auf Menschenrechte im Einklang mit den durch die Konvention vorgegebenen Standards prägt. Dies gilt in besonderem Maße für die strafprozessualen Garantien. Um den Einfluss der angegebenen internationalen Rechtsschutzmechanismen möglichst objektiv beurteilen zu können ist es notwendig, zunächst den rechtlichen Status der Konvention als internationales Abkommen im Bereich der Menschenrechte sowie der Rechtsprechung des EGMR zu betrachten.

\section{Rechtlicher Status der EMRK und der Rechtsprechung des EGMR im russischen Rechtssystem}

Dieser Teil gilt der Frage, inwieweit man von einer Priorität der Normen der internationalen Verträge und ihrer direkten Anwendung sprechen kann, insbesondere was die in der Konvention verankerten Vorschriften und die von dem Europäischen Gerichtshof entwickelten Standards betrifft.

\section{Rechtlicher Status der Konvention in der russischen Rechtsordnung}

Der Status des Völkerrechts im allgemeinen und der Konvention im besonderen wird im russischen Recht durch die Verfassung, entsprechende Gesetze, durch die Entschei-

13 S. auch S. Paramonova, Principle of Legality in Russia, in: U. Sieber/S. Forster/K. Jarvers (Hrsg.), National Criminal Law in a Comparative Legal Context, Vol. 2.1: General limitations on the application of criminal law, Berlin 2011, S. $103 \mathrm{ff}$.

14 Strafprozessordnung der Russischen Föderation vom 18. Dezember 2001, N 174-FZ, i.Kr. 1. Juli 2002, Ugolovno-prozessualnij Kodeks, nachfolgend UPK.

15 I.E. Paramonova/Bogush (Fn. 4). 
dungen des Verfassungsgerichts der Russischen Föderation sowie durch die Anordnungen des Obersten Gerichts der Russischen Föderation, bestimmt.

\section{a) Vorschriften der Verfassung über den Status der Konvention und ihre Interpretation}

Eine entscheidende Rolle bei der Bestimmung des rechtlichen Status der Konvention als internationales Abkommen spielt die russische Verfassung: Erstens enthält sie die menschenrechtlichen Grundsätze, zweitens die Regeln für die Anwendung des Völkerrechts.

\section{(1) Menschenrechtliche Grundsätze}

Zum einen spiegeln Art. 2 und Art. 18 Verfassung die Pflicht wieder, die in Art. 1 der Konvention festgelegt ist: jedem, der unter der Zuständigkeit der Russischen Föderation steht, Rechte und Freiheiten zu gewährleisten. Art. 2 Verfassung sieht vor: „Der Mensch, seine Rechte und Freiheiten bilden die höchsten Werte. Anerkennung, Wahrung und Schutz der Menschenrechte sind Verpflichtung des Staates“. Durch Art. 18 Verfassung ist eine direkte Anwendung der im Kapitel 2 Verfassung verankerten Rechte und Freiheiten garantiert: „Die Rechte und Freiheiten des Menschen und Bürgers gelten unmittelbar. Sie bestimmen den Sinn, den Inhalt und die Anwendung der Gesetze, die Tätigkeit der gesetzgebenden und der vollziehenden Gewalt sowie der örtlichen Selbstverwaltung und werden durch die Rechtsprechung gewährleistet". Zum anderen enthält Kapitel 2 der Verfassung über die „Rechte und Freiheiten des Menschen und Bürgers“ eine Liste von Rechten und Freiheiten, einschließlich der von der Konvention geschützten (Artikel 17-64 Verfassung). ${ }^{16}$ Damit sind die EMRK-Garantien kraft entsprechender verfassungsrechtlicher Bestimmungen in der russischen Rechtsordnung anwendbar.

\section{(2) Regelungen über die Anwendung des Völkerrechts}

Zum ersten Mal in der russischen Rechtsgeschichte wurde in Art. 15 Abs. 4 Verfassung der Grundsatz der direkten Anwendung der Regeln des Völkerrechts in der nationalen Rechtsordnung festgelegt: „Die allgemein anerkannten Prinzipien und Normen des Völkerrechts und die völkerrechtlichen Verträge der Russischen Föderation sind Bestandteil ihres Rechtssystems. Legt ein völkerrechtlicher Vertrag der Russischen Föderation andere Regeln fest als die gesetzlich vorgesehenen, so werden die Regeln des völkerrechtlichen Vertrages angewandt". Für die Analyse dieser Norm sollte man zunächst den von Art. 15 Abs. 4 Verfassung verwendeten Begriff ,,russisches Rechtssystem“ in Betracht ziehen. Inhaltlich gesehen ist dieser Begriff weiter als ,russische Gesetzgebung“". Das impliziert den Einfluss des Völkerrechts neben dem legislativen Bereich auf verschiedenen anderen Ebenen: die Regelungen der für Russland bindenden völkerrechtlichen Verträge (einschließlich der Konvention) sind in der Doktrin zu be-

16 S. Burkow (Fn. 10), S. 122 f. 
achten und im Prozess der Entwicklung neuer Gesetze sowie bei der Anwendung des Rechts bei den Strafverfolgungs- und Justizbehörden zu berücksichtigen. ${ }^{17}$

Von besonderer Bedeutung ist die Tatsache, dass das Prinzip der direkten Anwendung des Völkerrechts in Kapitel 1 Verfassung „Grundlagen der Verfassungsordnung“ verankert ist. Diese Platzierung hat folgende rechtliche Konsequenzen:

Erstens können keine Bestimmung der Verfassung oder andere rechtliche Normen Art. 15 Abs. 4 Verfassung widersprechen. Zweitens gehört dieses Prinzip zu den unveränderlichen Grundsätzen der Verfassung. Denn jede Änderung der Bestimmungen der Kapitel 1, 2 und 9 Verfassung setzt ihre Revision voraus, die eine Einberufung der Verfassungsversammlung bzw. die Durchführung einer Volksabstimmung fordert (Art. 135 Verfassung). Auf den ersten Blick legt also Art. 15 Abs. 4 Verfassung den unbestrittenen Vorrang internationaler Abkommen gegenüber nationalen Normen fest. Allerdings wirft eine nähere Analyse der Vorrangproblematik eine Reihe von Fragen auf. Zunächst gilt es herauszufinden, ob alle internationalen Verträge, insbesondere die EMRK, den Vorrang vor nationalen Gesetzen haben. Sodann ist zu entscheiden, ob die Verfassung selbst zu den nationalen Normen im Sinne des Art. 15 Abs. 4 Verfassung gehört und damit (im Bereich der Menschenrechte) den völkerrechtlichen Normen untergeordnet ist.

\section{(3) EMRK und nationale Gesetze}

In Bezug auf die erste Frage muss festgestellt werden, dass Art. 15 Abs. 4 Verfassung grundsätzlich vom Vorrang internationaler Abkommen gegenüber nationalen Normen ausgeht. In der Rechtslehre werden diesbezüglich sowohl monistische als auch dualistische Konzepte vertreten. Anhänger des monistischen Konzepts plädieren für die direkte Anwendung der Regeln der internationalen Abkommen, einschließlich der von Russland nicht ratifizierten Verträge. Ihrer Meinung zufolge sollten auch die Regeln, die nicht in das nationale Rechtssystem umgesetzt wurden, Vorrang vor nationalen Gesetzen haben. ${ }^{18}$ In Gesetzgebung und Lehre herrschend ist indessen ein dualistisches Konzept. Dem zufolge haben nur jene Bestimmungen der internationalen Verträge Vorrang gegenüber dem nationalen Recht, die in die russische Rechtsordnung entsprechend integriert wurden. ${ }^{19}$ Hinsichtlich der internationalen Menschenrechtsverträge ist die Ratifizierung eines Abkommens eine rechtlich geeignete Form der Umsetzung. ${ }^{20}$

In diesem Zusammenhang verdient Erwähnung, dass in der rechtswissenschaftlichen Doktrin vor allem der rechtliche Status der nicht ratifizierten internationalen Verträge

17 S. Marochkin, Deistvie norm mezhdunarodnogo prava v pravovoj sisteme RF, Tyumen 1998, S. 31.

18 S. Chernichenko, Sootnoschenie mezhdunarodnogo i wnutrigosudartvennogo prawa, Prawowedenie 2009, N 1, S. 18; J. Tichomirow/E. Talapina, Wwedenie v rossijskoe prawo, Moskau 2003, S. 50.

19 I. Lukaschuk, Russia's Conception of International Law, The Parker School Journal of East European Law 2 (1995), S. 14; V. Iwanenko, Mezhdunarodnie dogowori i konstituziya, Prawowedenie 2010, N 3, S. 135 ff.: s. für das Verfahren das Föderale Gesetz "Über internationale Abkommen" vom 15. Juli 1995, N 101-FZ; Entscheidung des Plenums des Obersten Gerichtshofs der RF vom 10. Oktober 2003, N 5.

20 Föderales Gesetz "Über internationale Abkommen"; Entscheidung des Plenums des Obersten Gerichtshofs der RF vom 10. Oktober 2003, N 5; Burkow (Fn. 10) S. 125; s. unten 2. 
gegenüber den nationalen Gesetzen umstritten ist. ${ }^{21}$ Im Hinblick darauf, dass ratifizierte internationale Verträge (d.h. jene Verträge, die offiziell gem. Art. 15 Abs. 3 Verfassung durch Erlass eines föderalen Gesetzes über die Ratifizierung veröffentlicht und damit als verfassungsgemäß anerkannt wurden) im Rahmen der Zuständigkeit der Russischen Föderation direkt und unmittelbar wirken, sind sich die meisten Wissenschaftler einig. Darunter fallen internationale Verträge, die strafprozessuale Garantien enthalten. ${ }^{22}$ Die EMRK, die die wesentlichen strafprozessualen Garantien verankert, trat für die Russische Föderation nach der offiziellen Veröffentlichung des Föderalen Gesetzes 1998 „Über die Ratifizierung der EMRK“23 in Kraft. Anders ist es beispielsweise mit internationalen Verträgen, die neue Straftatbestände einführen. Solche Vorschriften sogar ratifizierter internationaler Abkommen sollen durch entsprechende Änderungen des Strafgesetzbuches in das nationale Recht implementiert werden. ${ }^{24}$

Folglich hat die Konvention als ratifizierter internationaler Vertrag Vorrang gegenüber den Vorschriften des nationalen Rechts.

\section{(4) EMRK und die Verfassung}

Wenn man die zweite der oben gestellten Fragen bejaht (d.h. die Verfassung als nationales Gesetz im Sinne des Art. 15 Abs. 4 Verfassung anerkennt), kann man den Vorrang des Völkerrechts gegenüber den Verfassungsnormen bejahen. Doch ist das Problem des Vorranges der internationalen Abkommen gegenüber der Verfassung in Lehre und Praxis nicht vollständig gelöst. Dieses Phänomen wurde in der Doktrin „war of supremacies“" 25 getauft.

Einer der Gründe ist, dass die Normen der Verfassung selbst in dieser Hinsicht zweideutig sind. Einerseits sind gemäß Art. 15 Abs. 4 Verfassung die Bestimmungen des internationalen Vertrages anzuwenden, wenn ein internationaler Vertrag der Russischen Föderation vom Gesetz abweichende Bestimmungen enthält. Andererseits legt Art. 4 Abs. 2 Verfassung den Vorrang der Verfassung fest: „Die Verfassung der Russischen

21 Zum Protokoll Nr. 6 zur EMRK unten C); Iwanenko (Fn. 19), S. 135; W. Gavrilow, Mezhdunarodnaya i nazional'naya pravovaya sistema, Ponyatie I vsaimodeistvie mezhdunarodnoj I nazionalnoj pravovij system, Vladivostok 2005, S. 11; S. Bachin, Mezhdunarodnaya sostavlyauschaya pravovoi sistemi Rossii, Prawowedenie 2007, N 6, S. 126; Chernichenko (Fn. 18), S. 18; Tichomirow/Talapina (Fn. 18), S. 50.

22 Lukaschuk, (Fn. 19), S. 14; G. Danilenko, Implementation of International Law in CIS States: Theory and Practice, European Journal of International Law 10 (1999), S. 51 (53); Burkow (Fn. 10), S. 124.

23 Föderales Gesetz „Über die Ratifizierung der EMRK“ vom 30. März 1998, N 54-FZ.

24 Zum besonderen Status der internationalen Abkommen in Bezug auf strafbares Verhalten siehe Paramonova (Fn. 13) S. 105 ff. dies., Concept and systematization of the criminal offense in Russia, in: U. Sieber/S. Forster/K. Jarvers (Hrsg.), National Criminal Law in a Comparative Legal Context, Vol. 3.1: Defining criminal conduct, Berlin 2011, S. 86 ff; B. Zimnenko, Mezhdunarodnoe pravo, www.lib.rus.ec; Entscheidung des Plenums des Obersten Gerichtshofs der RF vom 10. Oktober 2003, N 5.

25 Iwanenko, (Fn. 19), S. 140; zum dualistischen und monistischen Konzept s. S. Paramonova. Strafrechtliche Jurisdiktion in Cyberspace: Rechtslage in Deutschland, Russland und den USA. Diss. Springer-Verlag. Berlin/Heidelberg/New York, 2013, i.E. 
Föderation und die föderalen Gesetze haben auf dem gesamten Territorium der Russischen Föderation Vorrang“".

In der Doktrin sind bezüglich dieses Problems zwei Hauptrichtungen entstanden. Nach der Meinung der „Internationalisten“ (im Gegensatz zu den „Verfassungsrechtlern") ist die Verfassung als nationales Gesetz im Sinne des Art. 15 Abs. 4 Verfassung einzustufen, womit sie dem Völkerrecht untersteht. Daher ist das Völkerrecht an der Spitze der Hierarchie der Rechtsquellen zu platzieren. ${ }^{26}$ Zudem hat sich in der Doktrin eine Meinung herausgebildet, dass nach Art. 17 Abs. 1 Verfassung die Vorschriften der internationalen Menschenrechtsabkommen Vorrang gegenüber den Bestimmungen der Verfassung haben oder mit ihnen auf gleicher Stufe stehen. ${ }^{27}$ Gemäß Art. 17 Abs. 1 Verfassung werden in Russland, ,die Rechte und Freiheiten des Menschen und Bürgers entsprechend den allgemein anerkannten Prinzipien und Normen des Völkerrechts und in Übereinstimmung mit dieser Verfassung anerkannt und garantiert“.

Allerdings sollte an der Stelle erwähnt werden, dass aus folgenden Gründen der Widerspruch zwischen den menschenrechtlichen Vorschriften der Verfassung und der Konvention zwar nicht ausgeschlossen, aber doch unwahrscheinlich ist: Erstens, weil die Verfassung - wie schon oben angedeutet wurde - unter der Berücksichtigung und aufgrund der Bestimmungen der Konvention entworfen wurde. ${ }^{28}$ Als Folge enthält das Grundgesetz die EMRK-Garantien (Art. 17-64 Verfassung). Zweitens wurde die Konvention ratifiziert. Das Verfahren setzt gemäß dem föderalen Gesetz „Über die internationalen Abkommen" voraus, dass die Vorschriften des entsprechenden Abkommens vor Ratifizierung als grundsätzlich verfassungsgemäß anerkannt werden. ${ }^{29}$

Ist es jedoch erforderlich, das Problem bezüglich dieses Vorrangs zu lösen, ist nach der hier vertretenen Auffassung dem Konzept der „Internationalisten“ unter Berücksichtigung der rechtlichen Regelung im Bereich der Menschenrechte zu folgen. Art. 18 Verfassung sieht vor, dass die Menschenrechte „unmittelbar gelten“ und „den Sinn, den Inhalt und die Anwendung der Gesetze, die Tätigkeit der gesetzgebenden und der vollziehenden Gewalt“ bestimmen ... und „durch die Rechtsprechung gewährleistet werden“. Art. 2 Verfassung erklärt Menschenrechte für „,den höchsten Wert“ und setzt damit den Vorrang der menschenrechtlichen Garantien gegenüber den anderen Verfassungsbestimmungen. Um den Vorrang der EMRK gegenüber den verfassungsrechtlichen Normen festzustellen, ist es folglich nicht erforderlich, das generelle Problem (Vorrang aller internationaler Abkommen vs. Verfassung als „nationales Gesetz“ im Sinne des Art. 15 Abs. 4 Verfassung) endgültig zu lösen. Aufgrund der Bestimmungen der Verfassung selbst (Art. 2 Verfassung i.V.m. Art. 15 Abs. 4 Verfassung) ist also vom Vorrang und von der direkten Wirkung der menschenrechtlichen Normen, die in den internationalen Abkommen festgelegt sind, auszugehen. Dieser Vorrang gilt sowohl gegenüber den nationalen (einschließlich Verfassungs-) Bestimmungen als auch gegenüber den Normen der internationalen Verträge, die direkt keine Menschenrechte verankern.

26 Iwanenko (Fn. 19), S. 135 ff.; Gavrilow (Fn. 21), S. 11; Bachin (Fn. 21), S. 126; Chernichenko (Fn. 18), S. 18.

27 I. Lukaschuk, Normi mezhdunarodnogo prava v pravovoj sisteme Rossii, Moskau 1997, S. $39 \mathrm{f}$.

28 S. oben 1. a.).

29 S. Föderales Gesetz “Über internationale Abkommen”, insbes. Art. 22. 


\section{b) Relevante Gesetze und höchste Gerichtsinstanzen über den Status der Konvention}

Der Status des Völkerrechts wird durch eine Reihe weiterer Gesetze bestimmt. Die Gesetze, die die wichtigsten strafprozessualen Garantien enthalten, legen einen Vorrang der internationalen Verträge vor den nationalen Normen fest: Art. 1 Strafprozessordnung sowie Art. 3 Strafvollzugsordnung. Das föderale Gesetz „Über internationale Verträge“" (in Art. 5 Abs. 2) geht ebenso wie die Verfassung (Art. 15 Abs. 4) von der Priorität der Normen der internationalen Verträge vor den nationalen Vorschriften aus. Das Gesetz unterscheidet zwischen zwei Gruppen von internationalen Verträgen.

Zur ersten Gruppe gehören solche Abkommen, die auch ohne den Erlass zusätzlicher nationaler Vorschriften eine direkte Wirkung innerhalb der russischen Rechtsordnung haben. Für die direkte Wirkung der Verträge der zweiten Gruppe sind entsprechende nationale Rechtsvorschriften notwendig, beispielsweise das Föderale Gesetz über die Ratifizierung (Art. 5 Abs. 3 Gesetzes). Die internationalen Abkommen der Russischen Föderation, „,deren Gegenstand Menschenrechte und Grundfreiheiten“ sind, unterliegen der obligatorischen Ratifizierung (Art. 15 Abs. 2 Gesetzes). Die Konvention, die die grundlegenden Menschenrechte und Grundfreiheiten verankert, gehört also zur zweiten Gruppe von internationalen Verträgen. Sie wurde gesetzesgemäß ratifiziert. Folglich hat die EMRK nach dem Gesetz „Über internationale Verträge“ eine direkte Wirkung und Vorrang gegenüber den nationalen Gesetzen.

Entscheidungen des Plenums des Obersten Gerichtshofs der Russischen Föderation gehen die spezifischen Probleme in einem bestimmten Bereich an und haben als Ziel, die Rechtsprechung in Bezug auf diese Fragen zu harmonisieren. Sie werden nicht als offizielle Rechtsquellen betrachtet. Dennoch gelten die Entscheidungen als de factoRechtsquellen: Gesetzlich sind Gerichte nur durch die Verfassung und föderale Gesetze gebunden, doch folgen sie den Entscheidungen konsequent in der Praxis (Art. 120 Verfassung). Die Entscheidung vom 2003 ist der Anwendung internationaler Abkommen bei den nationalen Gerichten gewidmet. ${ }^{30}$ Unter anderem wird in ihr auf die direkte und unmittelbare Wirkung und den Vorrang der ratifizierten internationalen Verträge vor den nationalen Normen hingewiesen, die strafprozessuale Normen enthalten. ${ }^{31}$ Jedoch lässt der Oberste Gerichtshof die Frage des Vorrangs der Konvention gegenüber der Verfassung unbeantwortet.

Das Verfassungsgericht betonte in seinen Entscheidungen mehrmals den Vorrang der Vorschriften der internationalen Verträge, insbesondere der EMRK und ihrer Zusatzprotokolle. ${ }^{32}$ Auf deren Priorität gegenüber der Verfassung wurde jedoch vom Verfassungsgericht nicht ausdrücklich eingegangen. Im Allgemeinen haben die Entscheidungen des Verfassungsgerichts normativen Charakter, da sie für eine unbestimmte Anzahl von Personen und in der Regel für unbestimmte Zeit gelten; sie sind nicht anfechtbar und treten sofort nach ihrer Verkündigung in Kraft. Daher haben auch solche Entscheidungen de facto die gleiche rechtliche Wirkung wie gesetzliche Bestimmungen. ${ }^{33}$

30 Entscheidung des Plenums des Obersten Gerichtshofs RF vom 10. Oktober 2003, N 5.

31 Ebd.

32 Entscheidung des Verfassungsgerichts RF vom 2. Februar 1996, N 4 П; vom 25. Januar 2001, N 1; vom 5. Februar 2007, N 2 П: http://www.ksrf.ru/Docs.

33 Entscheidung des Verfassungsgerichts RF vom 19. November 2009, N 1344-O-P über die Abschaffung der Todesstrafe in Russland. 


\section{c) Ergebnis}

Nach Analyse der gesetzlichen Ebene, der Verfassungsbestimmungen, der Lehre sowie der Rechtsprechung der höchsten Gerichtsinstanzen kommt man zum Schluss, dass der EMRK im Rahmen der Zuständigkeit der Russischen Föderation unmittelbare Wirkung zukommt. Nach der hier vertretenen Meinung hat die Konvention auch Vorrang vor allen nationalen Gesetzen, einschließlich der Verfassung.

\section{Rechtlicher Status der Praxis des EGMR in der russischen Rechtsordnung}

In Bezug auf den Status der Konvention bezüglich ihres Vorrangs gegenüber der Verfassung gibt es - wie oben gezeigt wurde - einige Meinungsverschiedenheiten. Jedoch treten keine ernsthaften rechtlichen oder dogmatischen Kontroversen auf, die eine direkte Anwendung ihrer Bestimmungen verhindern könnten. Hinsichtlich der direkten Anwendung der EGMR-Rechtsprechung im Rahmen des nationalen Rechtssystems ist die Situation nicht so eindeutig.

\section{a) Unterschiedliche Ansätze bezüglich des rechtlichen Status der EGMR-Rechtspre- chung}

Die Verfassung sowie die anderen Gesetze der Russischen Föderation enthalten keine ausdrücklichen Regelungen, die den rechtlichen Status der Rechtspraxis der internationalen rechtsprechenden Organe im nationalen Rechtssystem bestimmen. Das föderale Gesetz „Über internationale Verträge der Russischen Föderation“ führt folgende Subjekte auf, die für die Erfüllung der internationalen Abkommen Russlands zuständig sind: Präsident, Regierung, föderale Organe der exekutiven Gewalt und Staatsorgane der Subjekte der Russischen Föderation (der russischen Bundesländer). Jedoch werden in den Bestimmungen des Gesetzes zum einen nicht die Befugnisse der nationalen Gerichte erwähnt, die internationalen Abkommen anzuwenden, zum anderen bleibt der Status der Rechtsprechung internationaler Gerichte unbestimmt. Das könnte man dadurch erklären, dass dieses Gesetz nicht für die Umsetzung der internationalen Vereinbarungen vorgesehen wurde, nach denen Privatpersonen auf der internationalen Ebene vor Gericht die im jeweiligen Vertrag vorgesehenen Rechte durchsetzten können. Im föderalen Verfassungsgesetz von 1996 „Über das Justizsystem der Russischen Föderation“ wurde zum ersten Mal ausdrücklich die Anwendung der internationalen Regeln bei den nationalen Gerichten erwähnt. Dabei handelt es sich ausschließlich um die Anwendung der Normen und der ,,anerkannten Grundsätze“ des Völkerrechts. ${ }^{34}$

In der Doktrin haben sich verschiedene Ansätze bezüglich des Status der EGMRRechtsprechung herausgebildet. Im Allgemeinen unterscheiden sich die Konzepte nach dem Umfang des Anwendungsbereichs der gerichtlichen Praxis im Rahmen der nationalen Rechtsordnung. Die erste Ansicht spiegelt ein enges Konzept wieder. Es basiert auf der Tatsache, dass ein Präzedenzfall als solcher in einem kontinentalen Rechtssys-

34 Föderales Verfassungsgesetz vom 31. Dezember 1996, N 1-ФК3 „Über das Justizsystem der $R F^{*}$. 
tem (wie Russland) nicht als offizielle Rechtsquelle anerkannt ist. Daher könnten die EGMR-Entscheidungen keine Rechtsnormen kreieren. „Selbst wenn davon auszugehen ist, dass der EGMR Rechtsnormen schafft, sind diese nicht bindend für die Mitgliedstaaten, darunter Russland“. ${ }^{35}$ Dieser Ansatz beruht auf der Idee, dass für die souveränen Staaten nur der Text des jeweiligen obligatorischen Abkommens bindend ist, beispielsweise der EMRK-Normen. Auf solche Weise können die internationalen Normen ausschließlich von den Subjekten des Völkerrechts, den souveränen Staaten (reciprocity principle), geschaffen werden.

Diese Sichtweise wurde zu Recht kritisiert. Das Hauptziel der Konvention als Instrument für die Schaffung eines ordre public ist der Schutz der grundlegenden Rechte. Schließlich kann der klassische Grundsatz der Gegenseitigkeit nicht im Bereich der Europäischen Menschenrechte angewandt werden. ${ }^{36}$ Bereits im Jahre 1961 kam der EGMR in einer seiner Entscheidungen bezüglich der Zulässigkeit einer Klage von Österreich gegen Italien ${ }^{37}$ zu dem Schluss, dass der Zweck der Ratifizierung der EMRK bei den Mitgliedstaaten nicht war, gegenseitig Rechte und Pflichten abzugeben, um seine individuellen nationalen Interessen zu erreichen, sondern um die Ziele und Ideen des Europarates zu verwirklichen, die in der Konvention verankert sind, und um „eine gemeinsame öffentliche Ordnung (ordre public) der freien europäischen Demokratien zu etablieren". ${ }^{38}$ Darüber hinaus ist es praktisch unmöglich, den Sinn einer EMRKNorm lediglich auf der Grundlage des Textes der Konvention festzustellen. Denn die EGMR-Rechtsprechung entwickelt sich ständig weiter und füllt die Bestimmungen der EMRK mit neuen Inhalten.

Das zweite Konzept (formaler Ansatz) basiert auf der Differenzierung der EGMRRechtsprechung nach der rechtlichen Verbindlichkeit. Nach diesem Ansatz sind für Russland ausschließlich die EGMR-Entscheidungen rechtsverbindlich, die bezüglich oder mit Teilnahme Russlands getroffen wurden. Die weiteren EGMR-Entscheidungen sollten von der Russischen Föderation berücksichtigt werden, doch haben sie für Russland keine rechtlich bindende Kraft. ${ }^{39}$ Dieser Ansatz basiert auf den Bestimmungen der EMRK sowie auf den Normen des föderalen Gesetzes „Über die Ratifizierung der Konvention“" von 1998. So sind nach Art. 46 EMRK für einen Vertragsstaat nur die Urteile bezüglich der Rechtssachen bindend, in denen er Partei ist. Nach dem letzten Absatz des Art. 1 des föderalen Gesetzes „Über die Ratifizierung der EMRK“, erkennt die Russische Föderation gemäß Art. 46 der Konvention die Zuständigkeit des EGMR ipso facto ohne zusätzliches Abkommen in den Fragen der Auslegung und Anwendung der EMRK und ihrer Protokolle an.

Das dritte Konzept repräsentiert einen weiteren (inhaltlichen) Ansatz. Demgemäß sind nicht nur die Vorschriften der internationalen Verträge, sondern auch die Rechtsprechung der entsprechenden internationalen Organe (wenn ihre Kompetenz von Russ-

35 B. Zimnenko, Reschenija ESPCH i pravovaya sistema RF, Moskowskij Zhurnal mezhdunarodnogo prawa 2004, N 2, S. 79.

36 Burkow (Fn. 10), S. 127.

37 Oesterreich vs. Italien, $\mathrm{Nr} .788 / 60,1961$.

38 Yearbook of the European Convention on Human Rights 1961, Den Haag 1962, S. 138.

39 J. Kozheurow, O Postanowlenii Plenuma Werchownogo Suda RF N 5 ot 10.10.2003, Zhurnal mezhdunarodnogo chastnogo prawa 2004, N 1 (43), S. 9. 
land anerkannt wurde) im Rahmen der nationalen Rechtsordnung erforderlich. ${ }^{40}$ Somit sind - dieser Ansicht nach - auch Entscheidungen bezüglich anderer Länder für Russland bindend. Dieser Ansatz beruht auf der Auslegung des Art. 46 Abs. 3 i.V.m. Art. 15 Abs. 4 der Verfassung. Nach Art. 15 Abs. 4 sind ,die allgemein anerkannten Prinzipien und Normen des Völkerrechts und die völkerrechtlichen Verträge der Russischen Föderation“ als „Bestandteil ihres Rechtssystems“ anerkannt. Gemäß Art. 46 Abs. 3 Verfassung ist jeder ,,berechtigt, sich gemäß den völkerrechtlichen Verträgen der Russischen Föderation an die zwischenstaatlichen Organe zum Schutz der Menschenrechte und -freiheiten zu wenden, wenn alle bestehenden innerstaatlichen Mittel des Rechtsschutzes ausgeschöpft sind“. Das heißt, dass nach den verfassungsrechtlichen Normen das Recht auf gerichtlichen Rechtsschutz nicht durch nationale Vorschriften und rechtliche Mechanismen beschränkt ist. Damit kann ein Präzedenzfall als legitimer Mechanismus, der beim EGMR (dessen Zuständigkeit Russland anerkannt hat) in Bezug auf alle teilnehmenden Länder eingesetzt wird, auch im Rahmen der russischen Rechtsordnung ungehindert anerkannt werden.

Das Verfassungsgericht Russlands, das wesentlich zur Entwicklung des Prinzips der direkten Anwendung des Völkerrechts innerhalb des nationalen Rechtssystems beigetragen hat, folgt dem weiteren Ansatz. In einer Reihe seiner Entscheidungen hat das Verfassungsgericht Art. 15 Abs. 4 Verfassung im weiteren Sinne interpretiert, so dass die gesamte EGMR-Rechtsprechung (einschließlich der Urteile bezüglich anderer Länder) als legitime Rechtsquelle im Rahmen des russischen Rechtssystems zu verstehen ist. ${ }^{41}$ Hier sollte erwähnt werden, dass das Verfassungsgericht in vielen seiner Entscheidungen die EGMR-Urteile (nicht nur die bezüglich Russlands) anwendet und zitiert. ${ }^{42}$ In der Entscheidung 2007 hat das Verfassungsgericht festgestellt: „die EMRK sowie die EGMR-Entscheidungen - soweit sie den Inhalt der EMRK-Rechte und Freiheiten interpretieren, einschließlich des Rechts des fairen Zugangs zu den Gerichten sind ein integraler Bestandteil des russischen Rechtssystems und sollten daher von dem Gesetzgeber bei der Regulierung der sozialen Beziehungen und von den staatlichen Behörden bei der Anwendung einschlägiger Gesetze berücksichtigt werden“. ${ }^{43}$ Dadurch hat das Verfassungsgericht - im Gegensatz zu dem Gesetzgeber - eine bindende Rechtswirksamkeit der EGMR-Urteile im Rahmen des russischen Rechtssystems nicht nur auf die Fälle beschränkt, in denen die Russische Föderation Partei ist, sondern auf die ganze EGMR-Rechtsprechung erweitert.

Bei der Auslegung der Entscheidung des Obersten Gerichtshofs Russlands, die der Anwendung des Völkerrechts in der nationalen Rechtsordnung gewidmet ist, kommt man zu der Schlussfolgerung, dass auch der Oberste Gerichtshof dem weiteren Ansatz in Bezug auf die EGMR-Rechtsprechung als Rechtsquelle folgt. Doch erstens schränkt der Oberste Gerichtshof den Kreis der verpflichteten Subjekte auf die nationalen Gerichten ein, und zweitens verwendet er eher vorsichtige Formulierungen hinsichtlich der Verbindlichkeit der EGMR-Rechtsprechung. Somit erlegt der Oberste Gerichtshof den

40 Burkow (Fn. 10), S. 126 ff.; Danilenko (Fn. 22), S. 68.

41 Entscheidungen des Verfassungsgerichts RF vom 2. Februar 1996, N 4 П; vom 25. Januar 2001, N 1; vom 5. Februar 2007, N 2 П.

42 S. etwa Entscheidungen des Verfassungsgerichts RF vom 30. November 2000, N 15 П; vom 25. Januar 2001, N 1.

43 Entscheidung des Verfassungsgerichts RF vom 5. Februar 2007. 
nationalen Gerichten die Pflicht auf, die Normen der Konvention „unter Berücksichtigung der EGMR-Rechtsprechung“ mit dem Zweck anzuwenden, ,jegliche Verletzung der EMRK zu vermeiden“. ${ }^{44}$

Aufgrund der Analyse verschiedener Ansätze bezüglich des Umfangs der Verbindlichkeit der EGMR-Rechtsprechung innerhalb der russischen Rechtsordnung kann man sagen, dass sich das zweite Konzept (formaler Ansatz), das auf der Auslegung des Art. 46 Abs. 1 EMRK beruht, von der Perspektive des positiven Rechts aus am besten begründen lässt. Doch scheint es, dass dieses Konzept zu einer effektiveren Verwirklichung des wichtigsten Ziels der Konvention (Einhaltung und Gewährleistung der Menschenrechte) nur in Verbindung mit dem dritten (inhaltlichen) Konzept beitragen kann, aus folgenden Gründen: Die Gestaltung der Rechtsprechung des EGMR hinsichtlich der Anwendung der Konvention begann lange bevor Russland dem Europarat beigetreten ist und die Konvention ratifiziert hat. Daher enthalten logischerweise die Entscheidungen, die bezüglich Russlands getroffen wurden, nicht alle vom Gerichtshof entwickelten Rechtspositionen und Prinzipien. Die Entscheidungen bezüglich Russlands beruhen auf den Ansätzen, Konzepten und Kriterien, die vom EGMR in ähnlichen Fällen gegen andere Staaten ausgearbeitet wurden. Darüber hinaus entsteht durch die Anwendung der auf andere Staaten bezogenen EGMR-Rechtsprechung in Russland kein Widerspruch zum innerstaatlichen System der legitimen Rechtsquellen, insbesondere hinsichtlich der Einstufung der Rechtsprechung als de facto-Rechtsquelle. So gelten auch die Entscheidungen der obersten Gerichte, beispielsweise des Obersten Gerichtshofs RF, in Russland als de facto-Rechtsquellen. ${ }^{45}$ In der Praxis folgen Richter, die formal nur dem Gesetz und der Verfassung unterstellt sind (Art. 120 Abs. 1 Verfassung), konsequent den Entscheidungen der höheren Instanzen. Die Entscheidungen des Verfassungsgerichts haben, wie oben erwähnt wurde, de facto einen normativen Charakter. ${ }^{46}$

\section{b) Ergebnis}

Folglich sind zwar streng betrachtet ausschließlich die EGMR-Entscheidungen für Russland rechtlich verbindlich (bezüglich Entschädigungsvorschriften sowie Rechtspositionen des EGMR), in deren Rahmen es als Partei auftritt. Nach der hier vertretenen Auffassung sollen aber ebenso die bezüglich anderer Mitgliedstaaten getroffenen EGMR-Entscheidungen von allen staatlichen Gewalten Russlands, insbesondere von der Judikative, berücksichtigt werden. ${ }^{47}$ Eine solche ,,präventive Beachtung“ dient der Anwendung der nationalen Gesetze in Übereinstimmung mit der Konvention und ermöglicht es, in der Zukunft Verletzungen der EMRK zu vermeiden. ${ }^{48}$

44 Entscheidung des Plenums des Obersten Gerichtshofs der RF vom 10. Oktober 2003, N 5.

45 S. oben 2.

46 S. oben 2.; III. 2. (Abschaffung der Todesstrafe de jure).

47 Entscheidung des Verfassungsgerichts RF vom 5. Februar 2007.

48 S. Bericht vom 1998 über die Anwendung der EMRK im russischen Rechtssystem „Konvenzija i zakonodatelstvo i pravoprimenitelnaja praktika v RF (sravnitelnij analis)“: Rossijskaja Gazeta vom 6. Januar 1997. 


\section{Rolle der Konvention und der Rechtsprechung der EGMR bei der Ge- staltung des russischen Strafprozesses}

In diesem Teil soll der Einfluss der Umsetzung der EMRK und ihrer Protokolle sowie der EGMR-Rechtsprechung auf bestimmte Aspekte des russischen Strafverfahrens aufgezeigt werden. Die Analyse des Einflusses wurde auf verschiedenen Ebenen durchgeführt, die im Folgenden getrennt durchgegangen werden.

\section{Die konzeptionelle Ebene}

Zum ersten Mal in der Geschichte des russischen Rechts wurde mit der Verabschiedung der Verfassung im Jahr 1993 der Vorrang des Völkerrechts vor nationalem Recht (Art. 15 Abs. 4 Verfassung) verankert. Dies schuf eine Rechtsgrundlage für den im Prinzip „revolutionären“49 Schritt - die EMRK zu ratifizieren und damit die Souveränität des Staates zu beschränken. Bei der Anerkennung der obligatorischen Zuständigkeit des EGMR haben die russischen Behörden den Bürgern ein wirksames Instrument in die Hände gegeben, ihre Rechte und Interessen gegen die Missbräuche des Staates zu schützen. Dieses rechtliche Instrument erlaubt es, die nicht EMRK-konformen nationalen Gesetze, insbesondere strafprozessuale Vorschriften, sowie rechtskräftige Urteile zu ändern.

Nach heutigem Recht gehören gemäß Art. 413 Teil 4 Abs. 2 i.V.m. Art. 415 Abs. 5 UPK die vom EGMR festgestellten Verletzungen der EMRK im Rahmen der nationalen Strafverhandlung zu den neuen Umständen, die die Aufhebung eines rechtskräftigen Urteils bewirken können. Solche Verletzungen sollen entweder mit der Anwendung eines föderalen Gesetzes, das mit den Vorschriften der EMRK nicht übereinstimmt, oder mit anderen Verletzungen der Konvention verbunden sein. Nach der entsprechenden Entscheidung des EGMR soll nicht nur das Urteil des nationalen Gerichts aufgehoben oder geändert, sondern auch nötige Änderungen in die strafprozessuale Gesetzgebung eingefügt werden. ${ }^{50}$

2. Die Ebene des direkten Einflusses der Konvention, ihrer Protokolle und der EMRKRechtsprechung auf die Gestaltung des nationalen Strafprozessrechts

In diesem Abschnitt werden ausgewählte Problemaspekte (ohne andere, etwa zahlreiche Verletzungen des Folterverbot des Art. 3 EMRK vernachlässigen zu wollen) und damit verbundene entsprechende Änderungen der nationalen strafprozessualen Normen analysiert, die vor allem durch die Normen der Konvention und der EGMR-Rechtsprechung ins Leben gerufen wurden. ${ }^{51}$ Seit 1959 verkündete der EGMR mehr als 15.000 Urteile. Ungefähr die Hälfte der Urteile betraf vier Mitgliedstaaten. Russland belegt in dieser

49 Mälksoo (Fn. 6), S. 350.

50 Paramonova/Bogush (Fn. 4); M. Hussner, Die Umsetzung von Art. 6 Abs. 3 EMRK in der neuen Strafprozessordnung Russlands, Berlin, 2008, S. 1 ff.

51 Für eine Analyse der weiteren Gesetzesänderungen durch den Einfluss der EGMR-Rechtsprechung, etwa im Bereich der Unabhängigkeit der Richter, s. Paramonova/Bogush (Fn. 4). 
Liste mit 1.212 Beschwerden den unehrenhaften dritten Platz. ${ }^{52}$ Ohne die Bedeutung anderer Normen und Institutionen des internationalen Rechts zu mindern, hat die Gerichtspraxis des EGMR den größten Einfluss auf die Gestaltung des Strafprozesses in Russland.

\section{a) Unangemessene Fristen des Strafverfahrens}

Das Problem der Überschreitung einer angemessenen Verfahrensdauer (Art. 6 EMRK, Recht auf ein faires Verfahren) ist Gegenstand der meisten Beschwerden russischer Bürger vor dem EGMR. ${ }^{53}$ Auf den systematischen Charakter dieses Problems sowohl im Straf- als auch im Zivilprozess wurde in mehreren Entscheidungen des EGMR hingewiesen. In diesen Urteilen kam der Gerichtshof zu dem Schluss, dass es in Russland keine wirksamen Rechtsmittel gegen übermäßige Fristen eines Ermittlungsverfahrens sowie eines Gerichtsverfahrens gibt. ${ }^{54}$ Es sollte erwähnt werden, dass im Vergleich zu den prozessualen ,generellen Maßnahmen“ (measures of a general nature) die individuellen prozessualen Maßnahmen, etwa die Entschädigung für Opfer, auf nationaler Ebene einen effektiveren Vollzugsmechanismus haben. Allerdings sind in Bezug auf die „generellen Maßnahmen“ gewisse Erfolge durch den positiven Einfluss der EGMRRechtsprechung zu erkennen.

Die erste Rechtssache, in der der EGMR eine Überschreitung einer angemessenen Strafverfahrensdauer feststellte, war der Fall Kalaschnikow v. Russland. ${ }^{55}$ In diesem Fall hat das Gericht seine Entscheidung auf das Prinzip der Berücksichtigung aller Umstände gegründet, das er zuvor im Fall Kudla v. Polen entwickelt hatte. So legt der EGMR im Kalaschnikow-Urteil fest, dass „die Angemessenheit der Verfahrensdauer in Anbetracht der konkreten Umstände des Einzelfalls zu beurteilen ist". Vor allem sollten die von der EGMR-Rechtsprechung entwickelten folgende Kriterien berücksichtigt werden: „die Komplexität des Falles, das Verhalten des Antragstellers sowie das Verhalten der zuständigen Behörden. Darüber hinaus sollte auch der Charakter des Prozesses, und welche Bedeutung er für den Antragsteller hatte, berücksichtigt werden" ${ }^{56}$ Im Kalaschnikow-Fall hat der EGMR entschieden, dass die Verhandlungsdauer vor einem erstinstanzlichen Gericht, die mehr als vier Jahre beträgt, eine Verletzung des Grundsatzes der angemessenen Verfahrensdauer (Art. 6 Abs. 1 EMRK) ist. Das Gericht hat festgestellt, dass die vierjährige Dauer des Verfahrens mit der Komplexität des vorliegenden Unterschlagungsfalls nicht korrelierte.

Im Fall Smirnowa v. Russland ${ }^{57}$ hat der EGMR eine Verletzung des Art. 6 Abs. 1 EMRK aufgrund einer Strafverfahrensdauer von neun Jahren festgestellt. Im Fall Kho-

52 EGMR, Statistik 1959-2011, www.echr.coe.int.

53 EGMR, Statistik 1959-2011, www.echr.coe.int.

54 Kalaschnikow v. Russland vom 15.7.2002, Nr. 47095/99; Smirnowa v. Russland vom 24.7.2003, Nr. 46133/99; Fedorow and Fedorowa v. Russland vom 13.10.2005, Nr. 31008/02; Khodoyorow v. Russland vom 8.11.2005, Nr. 18147/02; Kormachewa v. Russland vom 29.1.2004, Nr. 53084/99 etc.

55 Kalaschnikow v. Russland.

56 Kalaschnikow v. Russland, Rn. 125.

57 Smirnowa v. Russland. 
doyorow v. Russland ${ }^{58}$ hat er das Maß der unangemessenen Verfahrensdauer auf eigene Initiative angehoben. ${ }^{59}$ Eine natürliche Folge zahlreicher Verletzungen des Art. 6 Abs. 1 EMRK, wie etwa die ungerechtfertigte Nichtbearbeitung von Beschwerden durch Gerichte und Ermittlungsbehörden, war eine weitere Reihe der EGMR-Entscheidungen, in denen im Zusammenhang mit den Verletzungen von Art. 6 EMRK auf Verstöße gegen Art. 13 EMRK (Recht auf einen wirksamen innerstaatlichen Rechtsbehelf) erkannt wurde. $^{60}$

Der wichtigste Grund für Verletzungen in dieser Problemkategorie war lange das Fehlen entsprechender Gesetzgebung. Es gab keine Vorschriften, die es erlaubten, die Richter zur Verantwortung zu ziehen, die eine unangemessene Verzögerung des Verfahrens verursachten. Es fehlte das innerstaatliche Verfahren, das eine effiziente Bearbeitung von Klagen über eine Verletzung des Rechts auf eine angemessene Verfahrensdauer ermöglichte. Ein gewisser Durchbruch kam nach dem Fall Burdow vs. Russland.$^{61}$ Der EGMR hat in seiner Entscheidung vom 15.1.2009 angeordnet, dass Russland bis Mai 2010 ,generelle Maßnahmen“ treffen solle, damit in der nationalen Rechtsprechung die Praxis überlanger Verfahren nicht fortgesetzt wird. Als Reaktion auf diese Entscheidung sowie als Ergebnis der langjährigen konsequenten Rechtsprechung des EGMR wurden entsprechende gesetzliche Änderungen auf der nationalen Ebene eingeführt. Erstens wurde mit der neuen UPK-Norm als neues strafprozessuales Prinzip das Prinzip der angemessenen Frist des Strafverfahrens (Art. 6.1 UPK) verkündet. Im Grunde hat der Gesetzgeber im neuen Art. 6.1 UPK das erwähnte Prinzip der Berücksichtigung aller Umstände bei der Bestimmung der angemessenen Strafverfahrensfristen übernommen. Die entsprechenden Änderungen sind im Mai 2010 in Kraft getreten. ${ }^{62}$ Zweitens wurde das föderale Gesetz „Über die Entschädigung für die Verletzung des Rechts auf ein Verfahren innerhalb einer angemessenen Frist oder des Rechts auf den Vollzug einer gerichtlichen Entscheidung innerhalb einer angemessenen Frist" erlassen. ${ }^{63}$ Auch dieses Gesetz ist im Mai 2010 in Kraft getreten. Dadurch haben russische Bürger das Recht auf eine entsprechende Entschädigung erhalten. ${ }^{64}$ Unter anderem setzte der durch das Gesetz eingeführte Mechanismus voraus, dass die Höhe der Entschädigung durch zuständige nationale Gerichte ,unter Berücksichtigung der vom EGMR entwickelten Grundsätze der Angemessenheit und Gerechtigkeit“ bestimmt wird (Art. 2 des föderalen Gesetzes „Über die Entschädigung“).

58 Khodoyorow v. Russland.

59 Khodoyorow v. Russland, Rn. 209.

60 Fedorov and Fedorova v. Russland; Plaksin v. Russland vom 29.4.2004, Nr. 14949/02; Zimenko v. Russland vom 23.6.2005, Nr. 70190/01.

61 Burdow v. Russland vom 15.1.2009, Nr. 33509/04.

62 Föderales Gesetz vom 30. April, 2010, N 69-FZ.

63 Föderales Gesetz „Über die Entschädigung für die Verletzung des Rechts auf ein Verfahren oder das Recht auf den Vollzug einer gerichtlichen Entscheidung innerhalb einer angemessenen Frist" vom 30.4.2010, N 68-FZ.

64 Paramonova/Bogush (Fn. 4). 


\section{b) Dauer der Untersuchungshaft}

Trotz dieser gesetzlichen Änderungen bleibt das Problem der unangemessenen Verfahrensfristen in Bezug auf die Zeit der Untersuchungshaft und damit einer Verletzung des Art. 5 EMRK aktuell. Die häufige Praxis der Gerichte, übermäßig lange Untersuchungshaftzeiten mit dem Verhängen einer Freiheitsstrafe zu „decken“, stößt immer wieder auf die Kritik von Menschenrechtlern. Oft hängt eine solche Praxis mit dem Mangel an Beweisen im konkreten Fall und mit der Zurückhaltung der Richter bei der Erkennung auf Freispruch zusammen. Als der EGMR Fälle von Verstößen gegen die Untersuchungshaftzeiten behandelt hat, hat er die Qualität der diesbezüglichen russischen Rechtsvorschriften nicht beanstandet. Diese erlauben Untersuchungshaft ausschließlich dann einzusetzen, ,wenn es unmöglich ist, eine mildere Zwangsmaßnahme zu verhängen“. Bei der Verhängung der Untersuchungshaft muss der Richter die konkreten rechtlichen und faktischen Umstände, auf denen seine Entscheidung beruht, nennen (Art. 29 und Art. 108 UPK). Allerdings hat der EGMR mehrmals die nationalen Gerichte Russlands für die Verhängung der Untersuchungshaft ohne begründete Argumente und unter Verletzung von Verfahrensfristen kritisiert. ${ }^{65}$ Beispielsweise verbrachte im Fall Smirnowa v. Russland ${ }^{66}$ eine der Beschuldigten mehr als vier Jahre in Untersuchungshaft. Bei der Analyse von richterlichen Beschlüssen über die Anordnung der Untersuchungshaft ist der EGMR zu dem Schluss gekommen, dass die Akten keine klare Begründung der Notwendigkeit der Verhängung solcher Zwangsmaßnahme und keine detaillierte Beschreibung der Situation der Beschuldigten enthielten. Alternative (mildere) Zwangsmaßnahmen wurden nicht in Betracht gezogen. In einem ähnlichen Fall, Kljakhin vs. Russland, ${ }^{67}$ in dem die konkreten faktischen Gründe für eine dreijährige Inhaftierung ebenfalls nicht ersichtlich waren, war nur die Schwere der Beschuldigung der Grund für die Verhängung der Untersuchungshaft. Dies führte zur Feststellung einer Verletzung des Art. 5 Abs. 3 EMRK.

Folglich hat sich im Rahmen des russischen Strafverfahrens eine Praxis herausgebildet, nach der eine Zwangsmaßnahme, insbesondere eine Anordnung der Untersuchungshaft, mit einem schlichten Hinweis auf die entsprechende Rechtsgrundlage (oft ohne Präzisierung des konkreten Absatzes des jeweiligen Artikels) begründet wird. Dagegen fehlt eine Darstellung der konkreten Umstände des Falles und der Besonderheiten der Situation des Verdächtigen, die jedoch eine faktische Voraussetzung für die Anwendung dieser Maßnahme bilden (Art. 29 und Art. 108 Abs. 1 UPK). Einer der Gründe, der aus historischer Perspektive zur Entwicklung einer solchen Praxis beigetragen hat, ist das bis 2002 existierende außergerichtliche Verfahren für die Anordnung freiheitsbeschränkender Zwangsmaßnahmen wie Inhaftnahme, Verlängerung der Untersuchungshaft und Arrest. ${ }^{68}$ Bis 2002 war hierfür lediglich ein Beschluss der Staatsanwaltschaft (ohne gerichtliche Kontrolle) erforderlich. Dies hat dazu beigetragen, dass die

65 Kalaschnikow v. Russland; Kljachin v. Russland vom 30.11.2004, Nr. 46082/99; Smirnowa v. Russland; Gusinskij v. Russland vom 19.5.2004, Nr. 70276/01 etc.

66 Smirnowa v. Russland.

67 Kljachin v. Russland.

68 Föderales Gesetz „Über das Inkrafttreten der Strafprozessordnung der RF“vom 18. Dezember 2001, N 177-FZ, i. Kr. 1. Januar 2004; Entscheidung des Verfassungsgerichts RF vom 14. März 2002, N 6 П. 
später eingeführte gerichtliche Kontrolle bei den Richtern als rein formales Instrument verstanden und von den praktizierenden Juristen auch so angewendet wurde. Im Jahr 2002 wurde die Befugnis, freiheitsbeschränkende Zwangsmaßnahmen anzuordnen, an die Gerichte übertragen. ${ }^{69}$

Im Hinblick auf diese Änderungen sollte man die entscheidende Rolle der EMRKBestimmungen betonen. Die neuen nationalen Vorschriften bezüglich der gerichtlichen Kontrolle im oben beschriebenen Zusammenhang wurden aufgrund der Konvention früher als vom Gesetzgeber ursprünglich vorgesehen eingeführt. ${ }^{70}$ Das Verfassungsgericht Russlands erklärte in seiner Entscheidung von $2002^{71}$ die Aufrechterhaltung des außergerichtlichen Verfahrens bis 2004 (wie ursprünglich vom entsprechenden Änderungsgesetz ${ }^{72}$ festgelegt) in Bezug auf strafprozessuale freiheitsbeschränkende Zwangsmaßnahmen für verfassungswidrig. Der Ausgangpunkt der Argumentation des Verfassungsgerichts war der Hinweis auf die Verletzung des Art. 5 Abs. 3 und 4 EMRK, und zwar des Rechts jeder Person, die festgenommen oder der die Freiheit entzogen ist, unverzüglich einem Richter vorgeführt zu werden. ${ }^{73}$

Obwohl sich der Anteil der vom Gericht genehmigten Anträge auf Verhaftung verringerte, bleibt dieser aber weiterhin unannehmbar hoch. Jedoch sind hier auch positive Änderungen zu sehen. Im Jahr 2010 ist die Novelle zur Strafprozessordnung in Kraft getreten, nach der eine Untersuchungshaft als Zwangsmaßnahme nicht gegen Personen verhängt werden kann, die wegen Wirtschaftsstraftaten angeklagt sind (Art. 108 Abs. $1.1 \mathrm{UPK}) .{ }^{74}$

Wie oben schon angedeutet, hängt die richterliche Praxis, lange Untersuchungshaftzeiten mit dem Verhängen einer Freiheitsstrafe zu „decken“, oft mit der Zurückhaltung der Richter zur Erkennung auf Freispruch zusammen. In der Tat ist die geringe Zahl der Freisprüche ein wichtiger Indikator dafür, dass die Gerichtspraxis extrem repressiven Charakter hat und eine unabhängige Justiz nach wie vor ein offener Wunsch bleibt. Laut statistischen Angaben beträgt die Freispruchrate (mit Ausnahme derjenigen der Jury-

69 Föderales Gesetz vom 18. Dezember 2001, N 177-FZ; Entscheidung des Verfassungsgerichts RF vom 14. März 2002, N 6 П.

70 Föderales Gesetz vom 18. Dezember 2001, N 177-FZ (Fn. 68).

71 Zur normativen Natur der Entscheidungen des Verfassungsgerichts s. oben II.1.b.

72 Art. 10 des Föderalen Gesetzes vom 18. Dezember 2001, N 177-FZ (Fn. 68). Gemäß dem Gesetz musste bis zum 1. Januar 2004 die Staatsanwaltschaft in Fragen des Verhängens freiheitsbeschränkender Zwangsmaßnahmen zuständig sein.

73 Entscheidung des Verfassungsgerichts RF vom 14. März 2002, N 6 П.

74 Föderales Gesetz vom 7. April 2010, N 60-FZ; S. Paramonova, Recent Reforms of Criminal and Criminal Procedural Law in Russia, Revista Penal 2012, N 30, S. 264 ff. 
gerichte, deren Zuständigkeitsbereich jedoch konsequent eingeschränkt wird ${ }^{75}$ ) weniger als $1 \% .{ }^{76}$

Das Problem ist unter anderem geschichtlichen Ursprungs. Der sowjetische Strafprozess zeichnete sich durch einen eindeutigen Inquisitions- (Fahndungs-) Charakter aus, Ermittler und Richter arbeiteten in allen Verfahrensstadien Hand in Hand. ${ }^{77}$ Auch in Bezug auf heute relevante Regelungen erregen einige Vorschriften Bedenken, die das Verhältnis zwischen Gericht und Staatsanwaltschaft im Zwischenverfahren betreffen. Art. 237 UPK erlaubt dem Gericht nach Erhalt der Anklageschrift und der Ermittlungsakte von der Staatsanwaltschaft, die die Grundlage der gerichtlichen Eröffnung des Verfahrens sind, die Ermittlungsakte vor der Eröffnung des Hauptverfahrens der Staatsanwaltschaft zurückzugeben. Gemäß Art. 237 UPK ist die Staatsanwaltschaft zur „Aufhebung der Hindernisse, die dem Gericht entstehen können, um über die Sache zu entscheiden“, verpflichtet. Zu diesen Hindernissen gehört es beispielsweise, wenn die Anklageschrift oder Ermittlungsakte unter Verstoß gegen die Vorschriften der Strafprozessordnung erstellt oder dem Angeschuldigten keine Kopie der Anklageschrift zur Verfügung gestellt wurde. Diese Regelung wirft einige Fragen auf. Aus Perspektive der Unabhängigkeit der Judikative, des Grundsatzes des fairen Gerichtsverfahrens und des Prinzips „,ne bis in idem“ ist es bedenklich, dass das Gericht und die Staatsanwaltschaft schon im Ermittlungsstadium zusammenarbeiten. Im Grunde erhält die Staatsanwaltschaft vom Gericht eine zweite Chance, die Anklageschrift nachzubessern. ${ }^{78}$ Letztlich ermittelt also der ganze staatliche Apparat gegen den Angeschuldigten. Man muss hier beachten, dass ein und dasselbe Gericht, das Ermittlungsakte und Anklageschrift bekommt und über die Zwangsmaßnahmen in der Sache entscheidet, auch für das Hauptverfahren zuständig ist. Eine solche Situation führt dazu, dass das Gericht, das zum „Redakteur“ der Anklageschrift wird, bei Entscheidung in der Sache kaum noch unvoreingenommen bleiben kann.

Ein weiterer Grund, der zur Entwicklung der Praxis des Einsatzes präventiver Maßnahmen unter Verletzung u.a. der Art. 5, 6 und 13 EMRK geführt hat, war das Fehlen jeglicher Kontrolle durch die höheren Gerichte. Dieser Mangel bezog sich sowohl auf Inhalt, Rechtmäßigkeit und Begründetheit der Entscheidungen der vorinstanzlichen Gerichte als auch auf Beschlüsse über die Anordnung von Zwangsmaßnahmen. Das Gericht zweiter Instanz hat in seiner Entscheidung in der Regel lediglich die Ergebnisse,

75 Eine breite Resonanz in der Öffentlichkeit hat das Föderale Gesetz vom 30. Dezember 2008 N 321-FZ ausgelöst, das den Zuständigkeitsbereich der Jury reduziert. Im UPK wurden entsprechende Änderungen (Art. 30 Abs. 2) eingeführt. Nach diesen bleibt eine Reihe von (nicht gerade politisch neutralen) Straftaten von der Gerichtsbarkeit der Jury ausgeschlossen. Dies sind beispielsweise folgende: Art. 205 UK „Terroristische Handlungen“, Art. 212 „Organisierung von Massenunruhen“, Art. 275 UK „Hochverrat“, Art. 279 UK „,bewaffneter Aufstand" etc. (insgesamt neun Straftatbestände).

76 Statistik der Gerichtsabteilung beim Obersten Gerichtshof, www.cdep.ru; S. Paramonova, Nowosibirsker Staatliche Universität (Hrsg.), Znachenie instituta suda prisjazhnich v rossijskoj sudebnoj sisteme / Aktuelle Probleme der geisteswissenschaftlichen und sozialen Forschungen, Novosibirsk 2011, S. 165 ff., auch zugänglich unter http://www.philosophy.nsc.ru/ youngcons/mol/2011.pdf.

77 Paramonova/Bogush (Fn. 4).

78 P. Lemke, Problemi ugolovnogo sudoproizvodsvtva v RF, Ugolovnoe sudoproizvodsvtvo 2010, N 4, S. $9 \mathrm{ff}$. 
zu denen das Gericht erster Instanz gekommen ist, wiederholt. ${ }^{79}$ Somit wurde auf problematische Stellen des Rechtsmittelsystems in Russland in mehreren EGMR-Entscheidungen hingewiesen. ${ }^{80}$

\section{c) Reform der Rechtsbehelfe}

Die Reform der Rechtsbehelfe, ${ }^{81}$ die 2010 begonnen hat, zählt zu den Maßnahmen, die unter dem Einfluss der Praxis des EGMR getroffen worden sind. Die letzten Änderungen sind 2013 in Kraft getreten. ${ }^{82}$

Das bisher geltende Rechtsmittelmodell sah zwei Instanzen vor: die Kassationsinstanz für die noch nicht rechtskräftigen Urteile (кассация) und die Aufsichtsinstanz für die in Kraft getretenen Urteile (надзор). Darüber hinaus hat sich in der Praxis eine eher formalistische Einstellung bezüglich des Kassationsverfahrens herausgebildet. Die Appellation (апелляция) konnte nur gegen die Urteile der Friedensrichter eingelegt werden. Dabei ist eine paradoxe Situation zu beobachten: einerseits war es möglich, die Appellation im Bezug auf Bagatelldelikte beim Bezirksgericht einzulegen (bei Straftaten, die in die Zuständigkeit des Friedensrichters fallen und mit maximal drei Jahren Freiheitsstrafe bedroht sind), andererseits gab es nur eine Kassation als Rechtsmittel bei schwerwiegenden Fällen. Die Aufsichtsinstanz als Instanz für die in Kraft getretenen Urteile löste viele Diskussionen im Hinblick auf das Prinzip res judicata (Art. 6 EMRK) aus und wurde vom EGMR nicht als wirksamer Rechtsbehelf anerkannt. ${ }^{83}$

Die neuen Änderungen lassen sich zwar nicht ohne Kritik, jedoch im Ganzen als positiv bewerten. Die Schaffung der vollwertigen Überprüfungsinstanzen könnte zu einer stärkeren Gewährleistung der Rechte und Interessen der Bürger beitragen. Beispielweise kann nach dem neuen Kapitel 45.1 UPK die Appellation gegen alle noch nicht rechtskräftigen Urteile und andere Entscheidungen der ersten Instanz eingelegt werden. Die Einlegung einer Aufsichtsklage wurde deutlich eingeschränkt. ${ }^{84}$

\section{d) Abschaffung der Todesstrafe}

Wer sich mit dem Einfluss der Konvention und ihrer Protokolle auf die russische Rechtsordnung im Allgemeinen und mit den nationalen strafprozessualen Vorschriften im Besonderen beschäftigt, kann kaum eine der wichtigsten Änderungen - die Abschaffung der Todesstrafe - umgehen. Auch wenn man die Meinung vertritt, dass die

79 A. Demenewa, Osnownie narushenija Evropeiskoj Konvenzii o zaschite prav cheloveka v RF, Ustanovlennie reschenijami Evpopejskogo suda po prawam cheloveka, http://echr-base.ru/ Publications/sut5/chapter3.pdf.

80 Ryabykh v. Russland vom 24. Juli 2003, Nr. 52854/99; Pravednaja v. Russland vom 18. November, 2004, Nr.69529/01; Wasiljew v. Russland vom 13. Oktober, 2005, Nr. 66543/01.

81 Detailliert zu der Reform der Rechtsbehelfe Paramonova/Bogush (Fn. 4).

82 Föderales Gesetz vom 29. Dezember 2010 N 433-FZ.

83 Ryabykh v. Russland; Pravednaja v. Russland; Smarigyn v. Russland vom 24. November 2005, Nr. 73203/01.

84 Paramonova/Bogush (Fn. 4). 
Abschaffung der Todesstrafe ein ausschließlich politisch bedingter Schritt Russlands für die Europaratsmitgliedschaft war, hat dieser Schritt doch vor allem nach der Ratifizierung der EMRK sowie des Protokolls Nr. 6 spürbare rechtliche und faktische Änderungen auf der nationalen Ebene bewirkt.

Wie bereits erwähnt wurde, gilt für die ratifizierten internationalen Abkommen, die die strafprozessualen Garantien verankern, der Grundsatz der direkten Anwendung innerhalb der nationalen Rechtsordnung. ${ }^{85}$ Umstritten ist der Status der nicht ratifizierten internationalen Verträge. Zu ihnen gehört beispielsweise das Protokoll Nr. 6 zur Abschaffung der Todesstrafe in Friedenszeiten. Es wurde von Russland unterzeichnet, aber nicht ratifiziert. Der herrschenden Meinung zufolge, die in der Lehre und in der Politik vertreten wird, wurde die Todesstrafe in Russland lediglich de facto abgeschafft. ${ }^{86} \mathrm{Nach}$ dem Standpunkt, der in diesem Beitrag vertreten wird, muss jedoch - wie die systematische Analyse der für Russland obligatorischen Normen völkerrechtlicher Verträge in Verbindung mit den einschlägigen nationalen Normen ergeben hat - von einer Abschaffung der Todesstrafe de jure die Rede sein. ${ }^{87}$ Als die Russische Föderation Mitglied des Europarates wurde und unmittelbar danach (1997) das Protokoll Nr. 6 EMRK unterzeichnete, verpflichtete sie sich, die erforderlichen Maßnahmen, die zu einer vollständigen Abschaffung der Todesstrafe führen, zu treffen. ${ }^{88}$ Ein de-facto Moratorium hatte es bereits im Jahre 1996 gegeben. Anschließend wurde die Todesstrafe ausdrücklich durch mehrere Entscheidungen des Verfassungsgerichts Russlands (die letzte vom Jahr 2009) suspendiert. ${ }^{89}$

Dennoch bleiben die der Todesstrafe gewidmeten nationalen Rechtsvorschriften nach wie vor in Kraft. Art. 20 der Verfassung, der die Todesstrafe als vorübergehende ,außerordentliche“ Strafmaßnahme vorsieht, erlaubt sie „bis zu ihrer Abschaffung“. In Übereinstimmung mit den verfassungsrechtlichen Normen sind im Strafgesetzbuch, in der Strafprozessordnung und in der Strafvollzugsordnung die entsprechenden materiellen und prozessualen Vorschriften für die Verhängung der Todesstrafe immer noch verankert. Beispielsweise wird nach dem Strafgesetzbuch die Todesstrafe als eine der verfügbaren Arten von Strafen (Art. 59 UK) angesehen. Das Strafgesetzbuch erlaubt sie für fünf „,besonders schwere Straftaten“ (Art. 15 UK) gegen das Leben. Daher ist eine Ambiguität bezüglich der Todesstrafe entstanden, denn einerseits bestehen nationale Rechtsvorschriften, die de jure immer noch die Anwendung der Todesstrafe erlauben, andererseits ist Russland internationalen Abkommen beigetreten, die die Anwendung der Todesstrafe verbieten.

85 S. oben II. 1. b.

86 Siehe z.B. A. Bystrikin (Leiter des Ermittlungsausschusses der RF): Russland ist noch nicht bereit, die Todesstrafe abzuschaffen (Russ.), 25.4.2013: http://www.rg.ru/2013/04/25/kaznanons.html; E. Archipowa, Problema otmeni smertnoj kazni v RF, 15.2.2012: http://www.scienceforum.ru/; A. Michlin, Wisschaja mera nakazaniya: istoriya, sovremennost, buduschee, Moskau 2000, S. 39.

87 S. Paramonova, Russia's Constitutional Court and the death penalty, in: L. Arroyo/P. Biglino/ W. Schabas (Hrsg.), Towards universal abolition of the death penalty, Valencia 2010, S. $167 \mathrm{ff}$.

88 Föderales Gesetz "Über den Beitritt der Russischen Föderation in den Europarat.

89 Entscheidungen des Verfassungsgerichts vom 2. Februar 1999, N 3-П; vom 19. November 2009, N 1344-O-P. 
Nach der Interpretation, die auf dem herrschenden dualistischen Konzept basiert, ist das Protokoll Nr. 6 für die Russische Föderation kein bindender Vertrag, bis es ratifiziert ist. Daraus folgt, dass die Todesstrafe noch nicht de jure verboten ist, da die Abschaffung der Todesstrafe auf nationaler Ebene lediglich durch die Entscheidungen des Verfassungsgerichts und nicht durch Rechtsnormen gewährleistet ist. Es scheint jedoch, dass diese Schlussfolgerung nicht alle Aspekte, die im Hinblick auf die internationalen Verpflichtungen hinsichtlich der Todesstrafe zu beachten sind, berücksichtigt. Nach der Unterzeichnung des Protokolls Nr. 6 zur EMRK hat sich Russland verpflichtet - wie es Art. 18 a der Wiener Übereinkommen über das Recht der Verträge fordert, wenn ein Staat einen Vertrag unterzeichnet hat - ,sich aller Handlungen zu enthalten, die Ziel und Zweck eines Vertrags vereiteln würde,... solange er seine Absicht nicht klar zu erkennen gegeben hat, nicht Vertragspartei zu werden“. ${ }^{90}$ Die Wiener Konvention wurde von Russland ratifiziert. Daher sind ihre Vorschriften für die Russische Föderation verbindlich. Das Protokoll Nr. 6 hat Russland noch nicht ratifiziert, jedoch wurde keine Absicht zum Ausdruck gebracht, nicht Vertragspartei daran zu werden. Das Protokoll hat folglich (als verbindlicher internationaler Vertrag) nach Art. 15 Abs. 4 Verfassung den Vorrang vor den inländischen Rechtsnormen. ${ }^{91}$

Zusammen mit den Normen der internationalen Verträge gibt es hier einen weiteren Punkt, der Beachtung verdient. Gemeint ist die Schlüsselentscheidung des Verfassungsgerichts bezüglich der Todesstrafe von 2009. ${ }^{92}$ Gemäß dieser Entscheidung darf die Todesstrafe auch nach Ablauf des Moratoriums, das in einer früheren Entscheidung des Verfassungsgerichts von 1999 verkündet wurde, ${ }^{93}$ nicht angewendet werden. Das Moratorium wäre ursprünglich am 1. Januar 2010 abgelaufen. Das Leitmotiv dieser Entscheidung war die Notwendigkeit, die nationale Regulierung bezüglich der Todesstrafe in Übereinstimmung mit den internationalen Verpflichtungen der Russischen Föderation (vor allem im Hinblick auf die Unterzeichnung des Protokolls Nr. 6 zur EMRK) zu bringen. Nach dem Standpunkt des Verfassungsgerichts erscheint die Anwendung der innerstaatlichen strafrechtlichen Bestimmungen hinsichtlich der Todesstrafe als nicht mehr möglich, da sie nicht mit den internationalen Normen übereinstimmen. Neben dem Verweis auf internationale Verpflichtungen hebt das Verfassungsgericht Art. 20 der Verfassung hervor, wonach die Todesstrafe nur eine vorübergehende Maßnahme ist. Das Gericht kam zu dem Schluss: Aufgrund des langfristigen Moratoriums für die Todesstrafe in Russland wurden feste Garantien der Nichtanwendung der Todesstrafe entwickelt. Es führt zur Etablierung einer ,legitimen verfassungsmäßigen Ordnung “94 und zu einem irreversiblen Prozess der endgültigen Abschaffung der Todesstrafe.

In Bezug auf die Entscheidungen des Verfassungsgerichtshofes kann man von einer quasi-normativen Natur ausgehen, da sie für eine unbestimmte Anzahl von Personen und in der Regel für unbestimmte Zeit gelten, nicht anfechtbar sind, alle verpflichten und sofort nach ihrer Verkündigung in Kraft treten. ${ }^{95}$ Daher haben sie de facto die glei-

90 Wiener Übereinkommen über das Recht der Verträge vom 23. Mai 1969, ratifiziert von Russland am 29. April 1986, http://treaties.un.org.

91 I. Lukashuk, Mezhdunarodnoe pravo, Moskau 2005, S. 236 f.

92 Entscheidung des Verfassungsgerichts vom 19. November 2009, N 1344-O-P.

93 Entscheidung des Verfassungsgerichts vom 2. Februar 1999, 3-П.

94 Entscheidung des Verfassungsgerichts vom 19. November 2009, N 1344-O-P.

95 S. oben II.2. 
che rechtliche Wirkung wie gesetzliche Bestimmungen. Im Hinblick auf die Entscheidung von 2009 bedeutet dies, dass auf nationaler Ebene das Ziel der de jure-Abschaffung der Todesstrafe durch eine „de facto-Lösung“ erreicht wurde.

\section{Schlussfolgerung}

Die Anwendung der Normen der Konvention sowie die Wirkungen der EGMR-Rechtsprechung im Rahmen des russischen nationalen Rechtssystems innerhalb der letzten 15 Jahre haben sich als effektive rechtliche Instrumente erwiesen. Heute kann man sagen, dass der rechtliche Mechanismus des EGMR sich zu einem der wirksamsten internationalen Mechanismen für den Schutz der Menschenrechte, insbesondere in Bezug auf strafprozessuale Garantien in Russland, entwickelt. Die in diesem Beitrag analysierten gesetzlichen Änderungen, die vor allem von den oben genannten Mechanismen ausgelöst wurden, reflektieren den langsamen Gestaltungsprozess auf den verschiedenen Ebenen, d.h. der konzeptuellen, praxisbezogenen sowie gesetzlichen Ebene. Jede Änderung im Rechtssystem hat ihre eigene „Dynamik“. Bestimmte Änderungen könnten schon durch die Verabschiedung entsprechender Normen eine effektive und sofortige unmittelbare Auswirkung im Rahmen des Rechtssystems haben, wie etwa die Abschaffung der Todesstrafe durch die Unterzeichnung des Protokolls Nr. 6 EMRK. Andere müssen über eine lange Zeit reifen, damit sie sich zu wirksamen menschenrechtlichen Garantien entwickeln. Sie gehören zu den größten Herausforderungen des Rechtssystems. Russland ist da keine Ausnahme. Der rechtliche Mechanismus des EGMR ist deshalb ein Mittel, das für die Gestaltung des russischen Strafprozesses kaum $\mathrm{zu}$ unterschätzen ist, weil er das Rechtssystem mit seinen tiefgreifenden Problemen konfrontiert und konsequente Lösungen anbietet. Nur steter Tropfen höhlt den Stein. 